\title{
Modeling astatine production in liquid lead-bismuth spallation targets
}

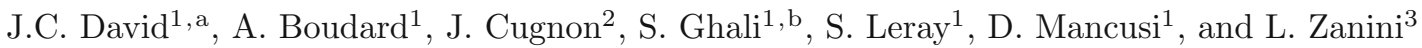 \\ 1 CEA/Saclay, Irfu/SPhN, 91191 Gif-sur-Yvette, Cedex, France \\ 2 University of Liège, AGO Department, allée du 6 août 17, Bât. B5, B-4000 Liège 1, Belgium \\ 3 ESS AB, P.O Box 176, SE-221 00 Lund, Sweden
}

Received: 21 December 2012 / Revised: 11 January 2013

Published online: 4 March 2013 - (C) Società Italiana di Fisica / Springer-Verlag 2013

Communicated by M. Hjorth-Jensen

\begin{abstract}
Astatine isotopes can be produced in liquid lead-bismuth eutectic targets through protoninduced double charge exchange reactions on bismuth or in secondary helium-induced interactions. Models implemented into the most common high-energy transport codes generally have difficulties to correctly estimate their production yields as was shown recently by the ISOLDE Collaboration, which measured release rates from a lead-bismuth target irradiated by 1.4 and $1 \mathrm{GeV}$ protons. In this paper, we first study the capability of the new version of the Liège intranuclear cascade model, INCL4.6, coupled to the deexcitation code ABLA07 to predict the different elementary reactions involved in the production of such isotopes through a detailed comparison of the model with the available experimental data from the literature. Although a few remaining deficiencies are identified, very satisfactory results are found, thanks in particular to improvements brought recently on the treatment of low-energy helium-induced reactions. The implementation of the models into MCNPX allows identifying the respective contributions of the different possible reaction channels in the ISOLDE case. Finally, the full simulation of the ISOLDE experiment is performed, taking into account the likely rather long diffusion time from the target, and compared with the measured diffusion rates for the different astatine isotopes, at the two studied energies, 1.4 and $1 \mathrm{GeV}$. The shape of the isotopic distribution is perfectly reproduced as well as the absolute release rates, assuming in the calculation a diffusion time between 5 and 10 hours. This work finally shows that our model, thanks to the attention paid to the emission of high-energy clusters and to low-energy cluster induced reactions, can be safely used within MCNPX to predict isotopes with a charge larger than that of the target by two units in spallation targets, and, probably, more generally to isotopes created in secondary reactions induced by composite particles.
\end{abstract}

\section{Introduction}

Spallation targets, generally made of heavy metal and bombarded by a high-intensity proton beam, can be used to produce intense neutron fluxes for condensed matter and material studies, in accelerator-driven sub-critical reactors, or in radioactive ion beam facilities. Liquid leadbismuth eutectic (LBE) is a possible choice for the target material and has been employed at the ISOLDE facility at CERN [1] and in the SINQ MEGAPIE target [2] at PSI. It is also the choice for the ADS demonstrator MYRRHA [3]. The spallation reactions occurring in the target give rise to a huge number of radioactive isotopes, some of which being volatile and therefore of particular concern in the case of liquid targets.

\footnotetext{
a e-mail: jean-christophe.david@cea.fr

b Present address: CEA/Saclay, DM2S/STMS/LGLS, 91191 Gif-sur-Yvette, Cedex, France.
}

Recently, the IS419 experiment at the ISOLDE facility at CERN measured the production and release rates of volatile elements from a LBE target irradiated by a proton beam of 1 and $1.4 \mathrm{GeV}[4,5]$. Among others, the production of At isotopes was investigated. Although the production of astatine isotopes is relatively modest and these isotopes are generally short-lived, they could pose a radioprotection issue since astatine being a halogen is highly volatile. Released astatine isotopes mostly $\beta$-decay to polonium isotopes, which are particularly radiotoxic since they are, especially ${ }^{208} \mathrm{Po},{ }^{209} \mathrm{Po}$ and ${ }^{210} \mathrm{Po}$, rather long-lived alpha emitters. Note that polonium isotopes are also directly produced inside the target, in fact in much larger quantities, including ${ }^{210} \mathrm{Po}$ through activation of ${ }^{209} \mathrm{Bi}$ by low-energy neutrons if the target is surrounded by a moderator, but they are not expected to be released at temperature of normal operation. 
In ref. [5], the experimental results were compared to simulations with the high-energy transport codes FLUKA [6] and MCNPX [7]. In the case of MCNPX, two different spallation models, actually two different combinations of intranuclear cascade and de-excitation models, were tried: INCL4-ABLA [8,9] and BertiniDresner [10,11]. None of the calculations were able to predict either the order of magnitude of the measured astatine production or the shape of the isotopic distribution.

From the modeling point of view, the production of astatine from bismuth is interesting since its atomic charge $(Z=85)$ is larger than that of bismuth by two units. This means that it can be produced only through $\left(p, \pi^{-}\right)$ reactions on bismuth or via secondary reactions involving (mainly) ${ }^{3} \mathrm{He}$ or ${ }^{4} \mathrm{He}$. Therefore, a reliable prediction of astatine yields by a given model is a test of its capability to correctly handle these particular channels.

Since the paper by Tall et al. [5], a lot of improvements have been brought to the INCL4-ABLA combination of models. New versions, INCL4.5 [12] and ABLA07 [13], respectively, have been developed and tested against an extensive set of experimental data, part of the work being done in the framework of the EUROTRANS/NUDATRA FP6 EC project [14], whose objective was to provide improved simulation tools for the design of ADS transmuters. The benchmark of spallation models organized under the auspices of IAEA [15] showed that this combination of models gives the best predictions of spallation residue production [16]. However, remaining deficiencies of INCL4.5 were identified, in particular concerning low incident energy and composite particle induced reactions, which had never been extensively tested. Therefore, and in particular in view of more reliable predictions of residues generated in secondary reactions, efforts have been devoted to improving the model on these points. The resulting version, called INCL4.6 is described in details elsewhere [17]. Coupled to the latest version of ABLA07, it has been implemented into a private version of MCNPX2.7b [18], thus allowing complete spallation targets simulations.

In this paper, after outlining the experimental results and briefly describing the model, we discuss its capability to reproduce the different reaction channels involved in the production of astatine in a $\mathrm{Pb}$ - $\mathrm{Bi}$ target. Then, we present the results of the simulation of the ISOLDE target irradiated with protons of 1.4 and $1 \mathrm{GeV}$ obtained with the model implemented into MCNPX and compare with the experimental data of ref. [5] on astatine release rates.

\section{The ISOLDE experiment}

The ISOLDE IS419 experiment was devoted to the study of the production and release rates of volatile elements from a liquid $\mathrm{Pb}-\mathrm{Bi}$ eutectic target irradiated with proton beams of 1.4 and $1 \mathrm{GeV}$. It is described in details in $[4,5,19]$. The lead-bismuth was contained in a tantalum cylinder of $20 \mathrm{~cm}$ length and $1 \mathrm{~cm}$ radius, $75 \%$ filled. The target temperature was fixed at $600^{\circ} \mathrm{C}$. The volatile elements produced in the liquid metal were ionized by means of a plasma ion source, then accelerated and sent

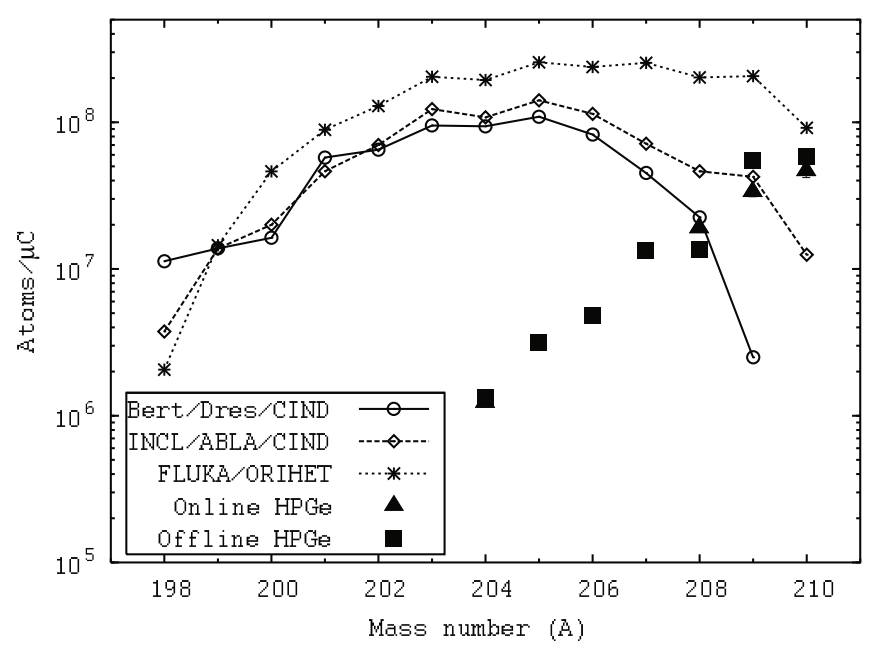

Fig. 1. Release rate of astatine isotopes from the ISOLDE LBE target at $1.4 \mathrm{GeV}$ measured by [5] and compared with the results of different transport codes (see text).

to a magnetic mass separators after which measurements were performed. Two different measurement techniques were used: off-line measurements using $\gamma$-spectroscopy are used for isotopes with half-lives from about 5 minutes to few weeks; on-line measurements for short-lived isotopes in which they are directed to a dedicated tape station where yields are measured.

The measured release rates are the results of complex processes since the volatile elements have to diffuse in the liquid metal, effuse through the target container, the transfer line and the ion source, be ionized, then accelerated and sent to the magnetic mass separators and to the beam lines where the measurements are done. The associated losses and efficiencies are rather well under control for noble gases and mercury. For astatine, measurements done at different temperatures [19] shows that at the target operation temperature, $600^{\circ} \mathrm{C}$, the release rate has reached a maximum, which seems to indicate that it is completely released. The diffusion time in the liquid metal also varies a lot depending on the element, for instance, it is a few minutes for noble gases but more than 30 min for mercury. For astatine, it is not known, which as will be shown in the following makes it more difficult to compare experiment and simulation.

The experimental isotopic distribution of released astatine is shown in fig. 1 extracted from ref. [5]. As explained above, the measurement was compared to calculations done with FLUKA [6] and MCNPX [7], in which two different spallation models were tried, INCL4.2-ABLA [8, 9] and Bertini-Dresner [10,11]. A complete and quasi instantaneous release was assumed. While some models were able to predict rather well noble gas and mercury release rates, it is striking that, besides the level of the rates that could be possibly explained by a smaller than expected release fraction, the shape of the measured isotopic distribution is totally different from the predicted ones, whatever the model used: the measured rates decrease with decreasing isotope mass while it increases or stays constant in 
the models. Clearly, efforts should be done to understand these features.

\section{The model}

Let us recall that spallation reactions are generally viewed as a two-step process: a first stage, the intranuclear cascade, in which the incoming nucleon experiences a series of nucleon-nucleon collisions leading to the ejection of fast particles and leaving an excited remnant, which, in a second stage de-excites through evaporation and sometimes fission. In this paper, we shall use the Liège intranuclear cascade model, INCL4 and for the de-excitation, the ABLA model developed in GSI, Darmstadt. A complete description of their initial version can be found in refs. [8] and [9], respectively. The first attempt to add the possibility of producing composite particles during the intranuclear cascade stage of INCL4, which, as will appear in the following, is very important for the purpose of this paper, was made in [20]. A lot of improvements were brought to the models which led to the versions tested in the IAEA benchmark, INCL4.5 [12] and ABLA07 [13]. However, the INCL4 version used in the present paper is INCL4.6, in which special attention has been paid to low incident energies and composite induced reactions, presented in details in [17]. Here, we just summarize the features of the model that are relevant for the production of $Z_{t}+2$ isotopes, i.e. isotopes with charge larger than the target one by 2 units.

As already mentioned, protons irradiating a $\mathrm{Pb} / \mathrm{Bi}$ target can produce astatine isotopes through the following mechanisms:

i) ${ }^{209} \mathrm{Bi}\left(p, \pi^{-} x n\right)^{210-x} \mathrm{At}$, i.e. double charge exchange in primary reactions, in which the proton is absorbed into the bismuth nucleus and a negative pion plus a certain number of neutrons (and possibly $\pi^{0}$ ) are emitted;

ii) $\left.{ }^{209} \mathrm{Bi}\left({ }^{3} \mathrm{He}, x n\right)\right)^{212-x} \mathrm{At}$ and $\left.{ }^{209} \mathrm{Bi}\left({ }^{4} \mathrm{He}, x n\right)\right)^{213-x} \mathrm{At}$, i.e. a two-step process helium-induced secondary reactions;

iii) secondary $\left(p, \pi^{-}\right),\left(d, \pi^{-}\right),\left(t, \pi^{-}\right),\left(\pi^{+}, \pi^{-}\right)$and reactions induced by ions heavier than helium can also lead to astatine isotopes but their contributions were checked to be negligible.

\subsection{Pion production}

In INCL4 pion production proceeds from the excitation of the $\Delta$ resonance. In the initial version [8], the model had a tendency to overpredict pion production cross-sections. In the INCL4.5 version, an average isospin-dependent potential well was introduced for pions, as well as reflection and/or transmission at the border of this potential. The phenomenological study that allowed to fix the depth of the potential is presented in detail in ref. [21]. The general result is a reduction of the pion production cross-sections, and a good agreement with experimental data, as illustrated by fig. 2 , in the case of negative pions.

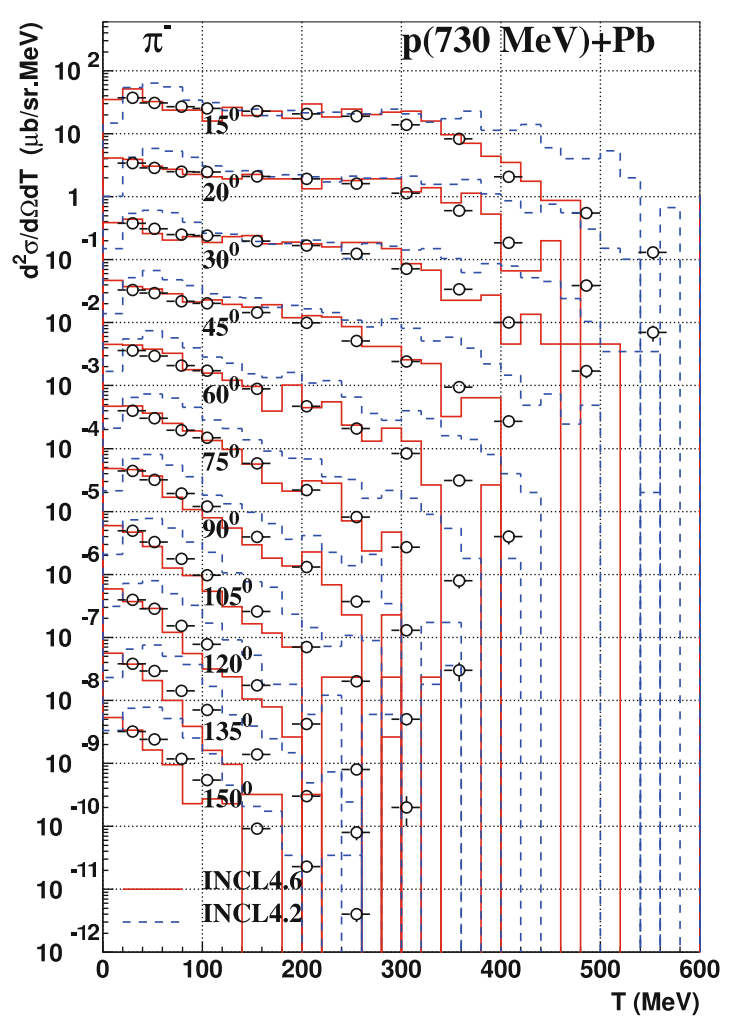

Fig. 2. Comparison of INCL4.2 (dashed blue lines) and INCL4.6 (red lines) predictions with experimental data for inclusive $\pi^{-}$double differential cross-sections in $p+{ }^{208} \mathrm{~Pb}$ collisions at $730 \mathrm{MeV}$ for different angles. Results at each angle are scaled down by a factor 10 starting from the smallest angle. Data are from ref. [22].

\subsection{Helium production}

Since secondary reactions of helium nuclei can be responsible for the production of astatine, it is important to have a model able to correctly predict helium production in spallation reactions. In particular, experimental helium energy spectra exhibit a high-energy tail that is often not accounted for by conventional spallation models since it cannot come from the evaporation stage and the INC emits only nucleons and pions. Only models having a specific mechanism to produce high-energy clusters of nucleons can aspire reproducing the experimentally observed high-energy tail. In INCL4 a mechanism based on surface coalescence in phase space has been introduced in ref. [20]. It assumes that a cascade nucleon ready to escape at the nuclear surface can coalesce with other nucleons close enough in phase space and form a cluster that will be emitted if its energy is sufficient to overcome the Coulomb barrier. All possible clusters are formed and the priority is given to the one with the lowest excitation energy per nucleon. In later versions of the models, the mechanism, originally limited to helium, has been extended to clusters up to mass 8 and the phenomenological parameters of the model, which include the volume of the phase space cell in which nucleons should be to form a cluster and the distance from the surface at which the clusters are 

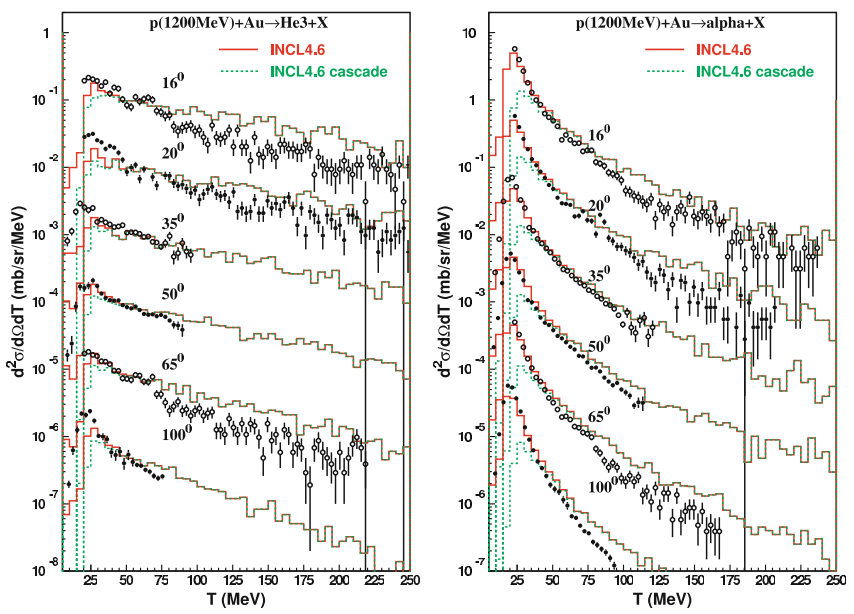

Fig. 3. Alpha and ${ }^{3} \mathrm{He}$ double-differential cross-sections, in the $p+\mathrm{Au}$ reaction at $1.2 \mathrm{GeV}$, measured by [23] (black dots), compared with the results of INCL4.6-ABLA07 (red lines). The cross-sections are mutliplied by 1, 10 and so on, successively, starting from the smallest angle. The contribution due to the coalescence process in the cascade is given by the dashed green line.

built, have been revisited. It should be stressed however that, like all the ingredients and parameters of INCL4, once chosen, the coalescence parameters are kept constant whatever the system studied.

In fig. 3, experimental data from [23] of alpha and ${ }^{3} \mathrm{He}$ production double-differential cross-sections, in the $p+\mathrm{Au}$ reaction at $1.2 \mathrm{GeV}$, are compared to the model predictions. A very good agreement can be observed all along the energy spectrum. In order to emphasize the importance of the coalescence mechanism the dashed green curve shows its contribution in the total production cross-sections. As already noticed in ref. [24], in the case of ${ }^{3} \mathrm{He}$, it represents the major part of the production cross-section.

\subsection{Helium-induced reactions}

The last feature that is very important in order to reliably predict astatine production is the treatment of the secondary helium-induced reactions. Although from the origin, the INCL4 model was designed to handle reactions with composite particle up to alpha, little attention had been paid to those up to recently. In addition, secondary reactions occur at low energies, generally below the alleged theoretical limit of validity of INC models. However, when models are implemented into transport codes and used for a large variety of applications, since no better solution exists (the data libraries available to the public transport codes do not consider yet reactions induced by complex particles), it is necessary to ensure the model predicts at least the gross features of all possible interactions. This is why an effort has been devoted to improve the treatment of low-energy composite particle induced reactions in INCL4. Details of the modifications brought to the model are discussed in [17]. Let us say here that the main improvements concern:

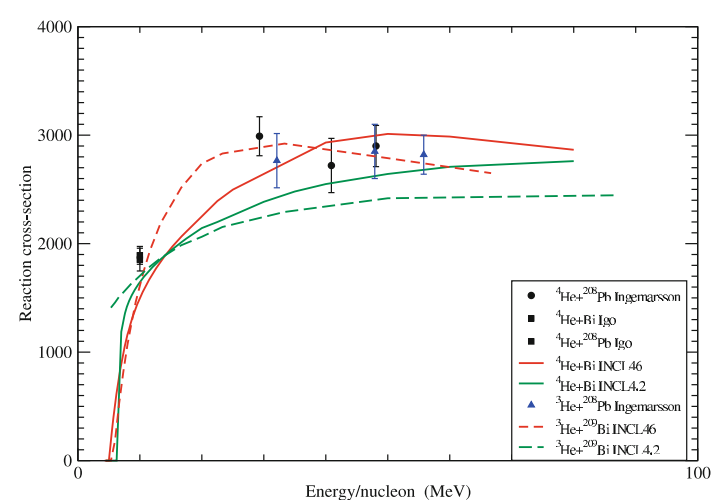

Fig. 4. Total reaction cross-sections as a function of incident energy for alphas (solid lines) and ${ }^{3} \mathrm{He}$ (dashed lines) on Bi calculated with INCL4.6-ABLA07 (red curves) and the older version INCL4.2-ABLA (green curves) compared to experimental data on $\mathrm{Pb}$ and $\mathrm{Bi}$ targets from [25-27].

a) the composite projectile, which was originally a collection of on-shell independent nucleons with internal Fermi motion superimposed to the collective motion, is now composed of off-shell nucleons bound by an ad hoc potential ensuring that the sum of the nucleon energies in the rest frame of the projectile is equal to the tabulated mass, therefore ensuring full energy and momentum conservation;

b) geometrical spectators, i.e. nucleons not passing through the target volume, are put on-shell and the energy needed to preserve a correct balance is taken from the participant nucleons;

c) if one of the nucleons tries to enter into the target below the Fermi level, all nucleons are absorbed leading to a total absorption of the projectile and the formation of a compound nucleus which is directly given to the de-excitation model;

d) the projectile Coulomb deviation is now explicitely taken into account;

e) experimental mass tables instead of average values, which were sufficient at high energies but were problematic close to reaction thresholds, are used, ensuring correct $Q$-values for the different reaction channels.

With these modifications, the model is able to predict rather well the helium-induced total reaction crosssections as can be seen in fig. 4, in which the model is compared to the few available experimental data [25-27] and to the predictions of the original version. This success may seem surprising since INC models are not supposed to be used to describe reactions below $\sim 150 \mathrm{MeV}$ and even less fusion reactions. Actually, this can be understood if one realizes that the model has by construction correct macroscopic properties, thanks to the realistic projectile and target densities and to the Coulomb deviation, and that, once nucleons have entered into the target nucleus, they are mostly trapped within the nuclear potential and not sensitive to the detail of the intranuclear cascade. The above-mentioned c) assumption goes also in this direction. This may be sufficient to get the gross features of fusion reactions. Our model obviously contains some 


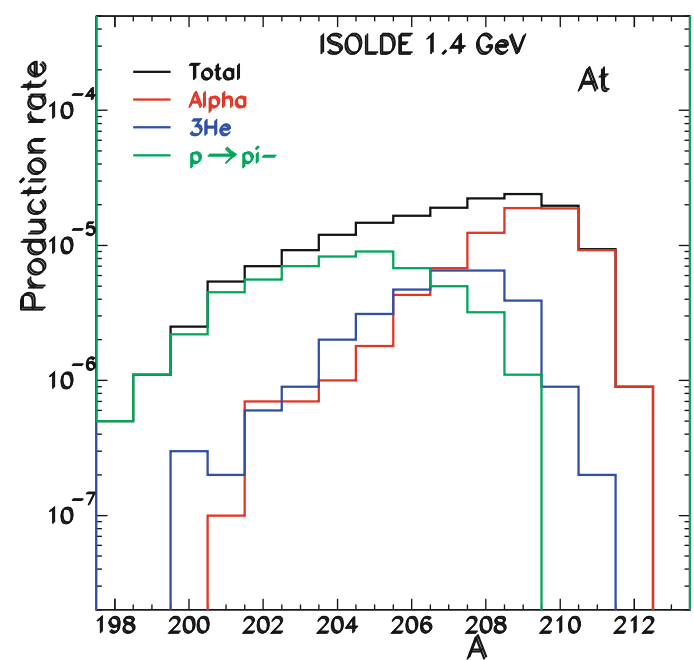

Fig. 5. Number of nuclei produced per incident proton for the different astatine isotopes as predicted by INCL4.6-ABLA07 implemented in MCNPX for the ISOLDE LBE target irradiated by $1.4 \mathrm{GeV}$ protons: black total production; green: production through $\left(p, \pi^{-}\right)$reactions; red and blue: through secondary reactions induced by alphas and ${ }^{3} \mathrm{He}$, respectively.

phenomenology but probably not more than many fusion models available on the market, with the advantage that, when the incident energy is increasing, the model can naturally describe the transition from complete to incomplete fusion. The predictions of the model concerning particular channels is discussed in the following section.

\section{Validation of the model for astatine production channels}

\subsection{Contributions of the different channels}

In the preceding section we have described the generic features of the model that are prerequisites for a correct prediction of astatine production channels, i.e. pion production, high-energy helium emission and low-energy helium interactions. Before going to a more detailed validation regarding the particular reaction channels involved in astatine production, let us estimate the level of the different contributions and the energies at which the reactions are occurring. The full simulation of the ISOLDE LBE target irradiated by $1.4 \mathrm{GeV}$ protons has therefore been performed using INCL4.6-ABLA07 implemented into MCNPX [18]. Figure 5 shows the global production rate per incident proton of the different astatine isotopes and the contributions from the different possible mechanisms: primary and secondary $\left(p, \pi^{-}\right)$reactions ( $46 \%$ of the total), secondary reactions induced by alphas (33\%) and ${ }^{3} \mathrm{He}(18 \%)$. It appears that the isotopes with mass larger than 209 are produced only through secondary heliuminduced reactions, which is not surprising since $\left(p, \pi^{-} x n\right)$ can lead only to $\mathrm{At}^{210-x}$. On the other hand, both mechanisms populate the other isotopes, the very lightest ones preferentially originating from double charge exchange

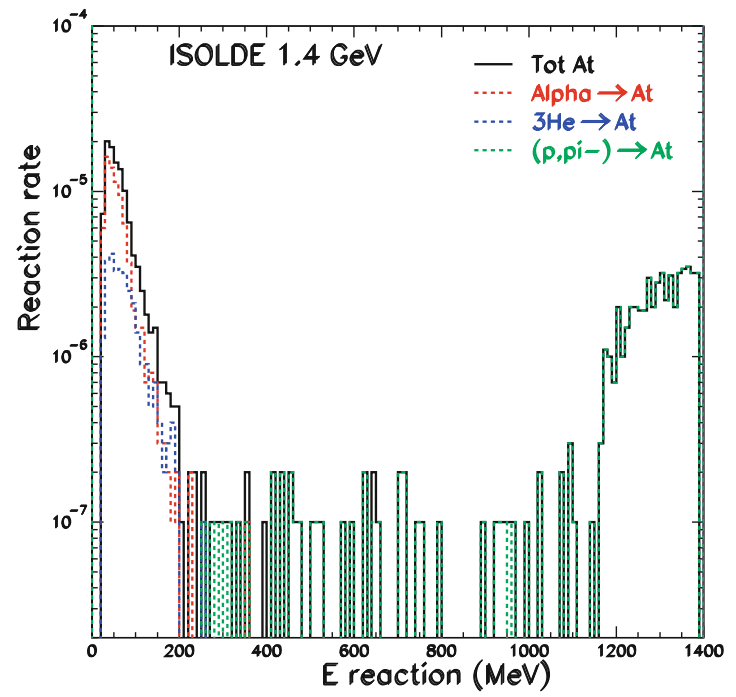

Fig. 6. Number per incident proton of reactions leading to astatine production, as a function of the energy at which the reaction occurs. Black: total; green: $\left(p, \pi^{-}\right)$reactions; red and blue: secondary reactions induced by alphas and ${ }^{3} \mathrm{He}$, respectively.

reactions. Regarding the respective contributions of ${ }^{3} \mathrm{He}-$ and ${ }^{4} \mathrm{He}$-induced reactions, it is found that alphas play a larger role and lead to higher masses, here also as could be expected.

The distribution of the incident kinetic energies at which the different reactions producing astatine isotopes occur is shown in fig. 6. It appears that double charge exchange reactions happen very close to the incident beam energy. This reflects the fact that the primary beam has a large probability to interact before slowing down and most of the primary reactions occur in the first centimeters of the target. Very few secondary proton reactions are observed. The spectrum of secondary helium-induced reactions presents a peak around $50 \mathrm{MeV}$ indicating that they are mostly provoked by nuclei originating from the cascade rather than from the evaporation stage, especially since they may have slowed down before interacting.

\subsection{Double charge exchange channel}

In sect. 3, it was shown that our model correctly predicts negative-pion production. However, what matters for astatine production is the particular channel of double charge exchange, in which a negative pion is emitted without any other charged particle. The population of this channel therefore also depends upon the probability to emit protons and clusters either in the cascade stage or during the de-excitation. The de-excitation itself is determined by the excitation energy at the end of the cascade stage and by the competition between the different particles that could be evaporated; fission could also play a role.

Unfortunately, very few thin-target experimental data are available to test our model on this particular channel. The only ones that are directly relevant to our case, 


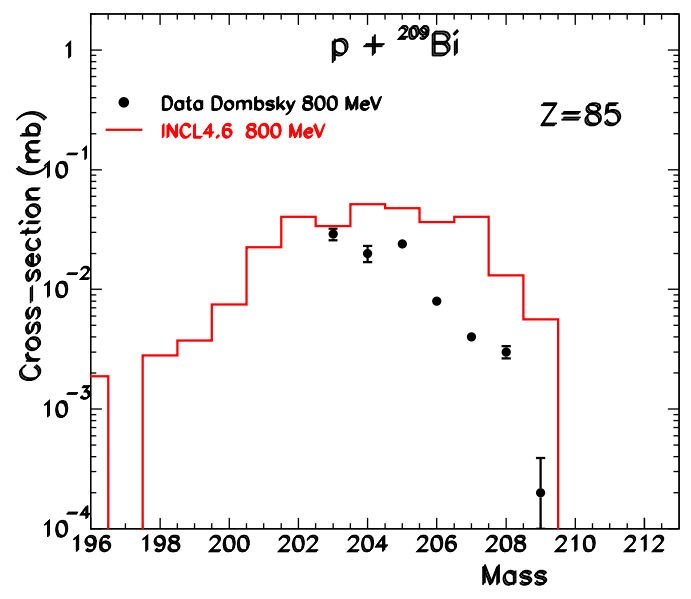

Fig. 7. Cross-sections for the production of different astatine isotopes from a Bi target irradiated by $800 \mathrm{MeV}$ protons measured by [28] and compared to the prediction of INCL4.6ABLA07.

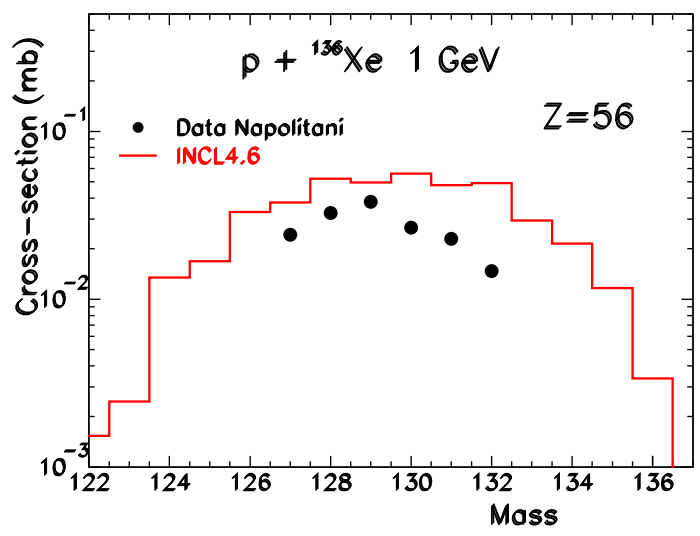

Fig. 8. Isotopic distribution of barium isotopes measured in reverse kinematics in the $p+{ }^{136} \mathrm{Xe}$ measured by Napolitani et al. [29] and compared to the prediction of INCL4.6-ABLA07.

i.e. for $\mathrm{Bi}$ at an incident energy around $1 \mathrm{GeV}$, are the data from Dombsky et al. [28] on $p+\mathrm{Bi}$ at $800 \mathrm{MeV}$ in which several astatine isotopes have been identified by radiochemical methods. They are displayed on fig. 7 together with the result of the calculation. In fig. 8, we also show isotopic distributions of $Z_{t}+2$ (barium) isotopes from the $p+{ }^{136} \mathrm{Xe}$ at $1 \mathrm{GeV}$ measured using the reverse kinematics method at GSI Darmstadt [29]. In both cases, the calculation overpredicts the experimental data by a factor 2 to 5 for the heaviest isotopes but less than 2 for the lightest ones. The reason for this is not well understood. It is probably linked to the ratio of charged particles versus neutrons emitted either in the evaporation or, more likely, during the cascade stage, since a larger emission of charged particle would depopulate the $Z_{t}+2$ production. Clearly, the responsibility of fission can be ruled out since both the Xe (which does not undergo fission) and Bi targets exhibits the same tendency.

However, since in the ISOLDE target the double charge exchange channel is a major contribution only for light isotopes, it can be expected that our simulation for those isotopes should be correct within a factor 2 with a tendency to an overprediction.

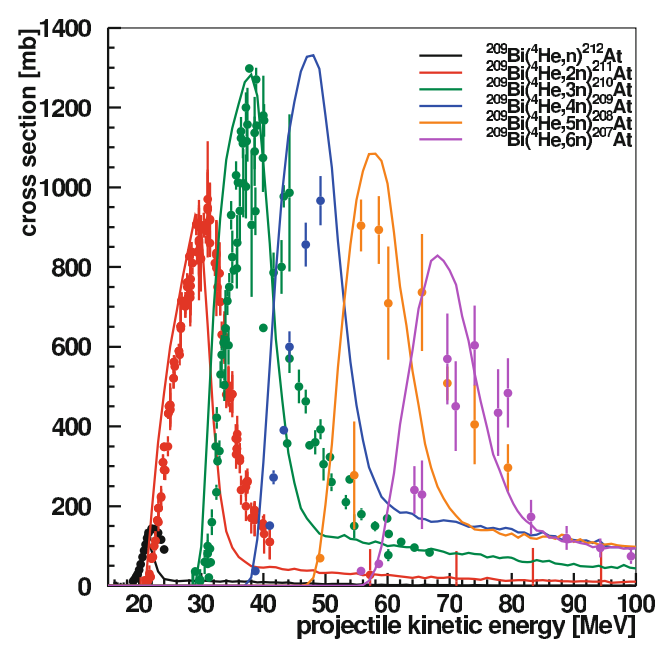

Fig. 9. ${ }^{209} \operatorname{Bi}(\alpha, x n)$ cross-sections for $x=1$ to 6 as functions of the $\alpha$ incident kinetic energy. The red curves correspond to the predictions of the INCL4.6+ABLA07 model. The experimental data were compiled using the experimental nuclear reaction database EXFOR [30].

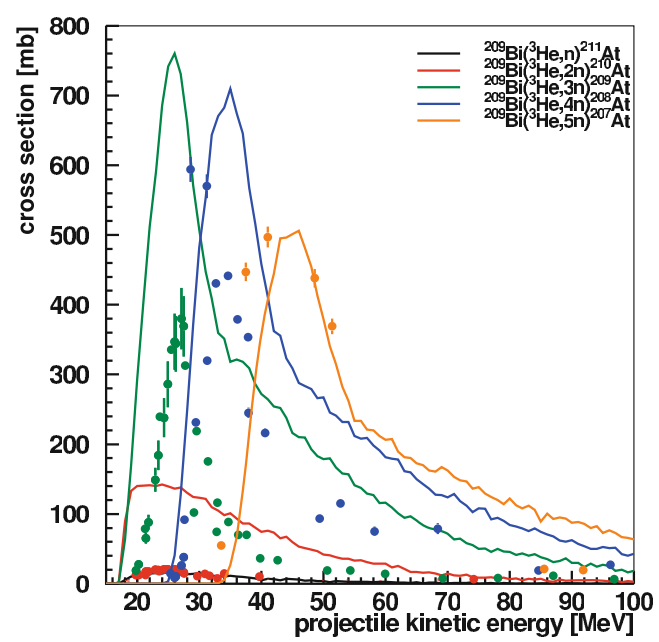

Fig. 10. Same as fig. 9 but for ${ }^{209} \mathrm{Bi}\left({ }^{3} \mathrm{He}, x n\right)$ cross-sections for $x=1$ to 5 .

\subsection{Secondary reaction channels}

The preceding sections have revealed that a major part of astatine is coming from secondary helium-induced reactions and that a priori our model correctly predicts the production of high-energy helium and the total reaction cross-sections at the energy at which the reactions take place. It remains to check that the particular $(\mathrm{He}, x n)$ channels involved in the prediction of the different isotopes are also under control.

In figs. 9 and 10, experimental cross-sections, found in the EXFOR experimental nuclear reaction database [30], regarding ${ }^{209} \mathrm{Bi}(\alpha, x n)$ and ${ }^{209} \mathrm{Bi}\left({ }^{3} \mathrm{He}, x n\right)$, respectively, for different $x$-values, as a function of the incident particle kinetic energy, are compared with the model. It can be observed that, for $\alpha$-induced reactions the model agrees 
rather well with the experimental results. In particular, the maximum cross-section for each channel is very well predicted. However, the opening and closing of the different channels as a function of incident energy seems to happen a little too early. This is probably related to the probability of emitting a given number of neutrons, which is very sensitive to model parameters used in the evaporation model such as the level densities or the inverse cross-sections. This idea is substantiated by tests done with another evaporation code, actually GEMINI $++[31$, 32 , which lead to a shift of the different channels as a function of energy [33]. The competition with proton emission is probably not relevant here either since, as observed in [34] in the similar case of a tantalum target, the sum of the different $(\alpha, x n)$ channels exhausts the total reaction cross-section.

In the case of ${ }^{3} \mathrm{He}$, the situation is less good, as can be seen in fig. 10 . The $x=1$ and $x=2$ channels are largely overestimated, the agreement being restored only for the largest $x$-values. The reason for this difference has been thoroughly investigated in [17], in particular by looking at the respective contributions from the cascade and evaporation stages in the different channels. Contrary to the ${ }^{4} \mathrm{He}$ case, in which the cascade contribution, except in the $1 n$ channel, is negligible close to the opening of the channel, for ${ }^{3} \mathrm{He}$ the $1 n, 2 n$ and to a lesser extent $3 n$ channels are mainly cascade populated. This is explained by the very different binding energies of ${ }^{3} \mathrm{He}$ and ${ }^{4} \mathrm{He}$ that has two consequences: first, the available energy after fusion, i.e. the incident energy plus the $Q$-value, is much larger in the case of ${ }^{3} \mathrm{He}$, meaning that several neutrons should be evaporated to exhaust the excitation energy and therefore the lowest $x$ channels cannot be populated by evaporation; second, ${ }^{3} \mathrm{He}$ being less bound has a higher probability to undergo a dissociation before reaching the target leading to one or two nucleons not entering the target or in other words to incomplete instead of complete fusion. This also explains that the sum of the different $\left({ }^{3} \mathrm{He}, x n\right)$ channels does not exhaust the total reaction cross-section contrary to the case of ${ }^{4} \mathrm{He}$, as observed in [34]. In our model, aspects linked to $Q$-values should be correctly taken into account, thanks to the fact that we now use experimental mass tables. On the contrary, the dissociation is mainly deriving from geometrical aspects (spectators) which may be not realistic enough. The large overprediction of the $x=1$ and $x=2$ channels and the fact that the associated neutrons are found to come from the cascade stage seems to indicate that the model does not predict enough absorption.

Actually, in the ISOLDE target secondary reactions induced by ${ }^{3} \mathrm{He}$ are much less numerous than by ${ }^{4} \mathrm{He}$ for the channels with the smallest $x$-values. Since our model agrees well with the experimental data for ${ }^{4} \mathrm{He}$ for all $x$-values and for ${ }^{3} \mathrm{He}$ for $x>2$, we can expect the overall prediction to be reliable within a factor definitely smaller than 2 . It should be stressed again that the intranuclear cascade is used here well beyond its alleged applicability limit and the overall agreement obtained in helium-induced reactions is globally very satisfactory.

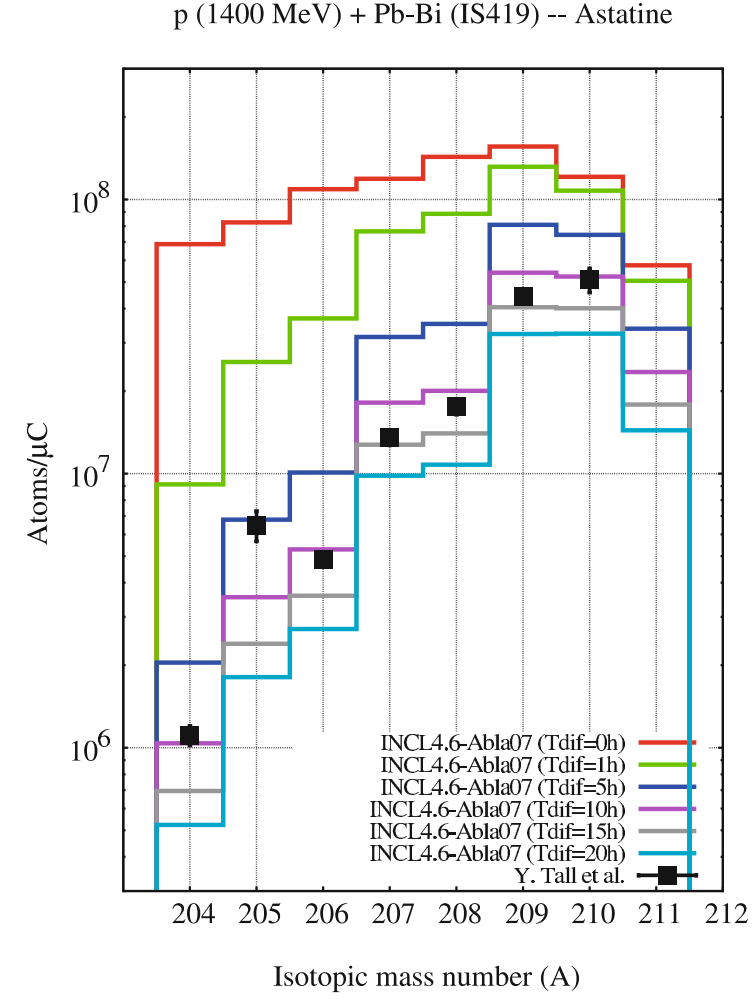

Fig. 11. Astatine release rates measured by the ISOLDE Collaboration [35] at $1.4 \mathrm{GeV}$ compared to MCNPX simulations, using INCL4.6-ABLA07. The solid red line is the yield given by MCNPX, the other lines are corrected to take into account the diffusion time and decay according to the formulas given in the appendix.

\section{Astatine production yields in the ISOLDE target}

\subsection{Influence of the diffusion time}

As mentioned above, the diffusion time of astatine in LBE is not known. In [5], the calculations were done assuming that this time is much smaller than the half-life of the measured astatine isotopes. If, on the contrary, this time is of the same order or larger than the half-life of some isotopes, these isotopes will partially decay before being released from the target. Actually, all the astatine isotopes have half-life shorter than or equal to $8 \mathrm{~h}$, some being much smaller, 9, 26 and 29 minutes for ${ }^{204} \mathrm{At},{ }^{205} \mathrm{At}$ and ${ }^{206} \mathrm{At}$, respectively. Moreover, astatine is a halogen and the release time of another halogen, iodine, has been measured in [19] and found to be of the order of several hours. This suggests that the question of the release time has to be carefully looked at.

A first simulation of the ISOLDE experiment has been done with INCL4.6-ABLA07 in MCNPX, assuming that the diffusion time is much smaller than the half-life of all measured isotopes, in which case the production yields can be directly compared to the experimental ones. The results of the calculations at 1.4 and $1 \mathrm{GeV}$ are compared to the experimental data in figs. 11 and 12 , respectively, as the 
$\mathrm{p}(1000 \mathrm{MeV})+\mathrm{Pb}-\mathrm{Bi}(\mathrm{IS} 419)--$ Astatine

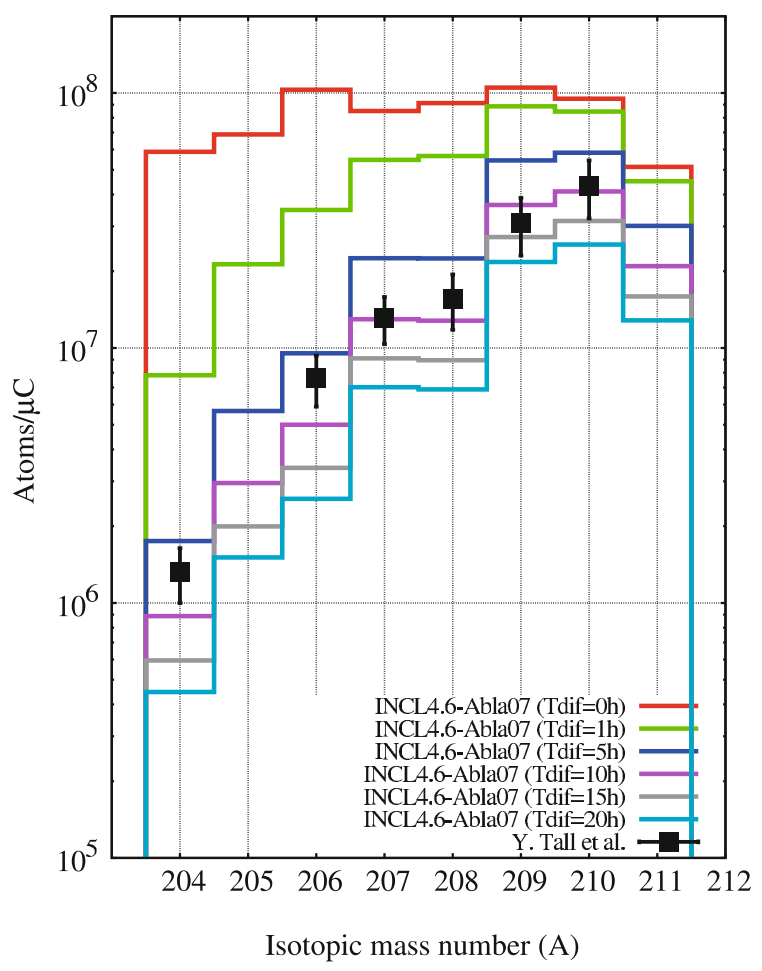

Fig. 12. Same as fig. 11 but at $1 \mathrm{GeV}$.

solid red curve. It should be noted that the experimental data presented here [35] are not exactly the same as in [5], in which they were still preliminary. Clearly, large differences between the calculation and the experimental data are observed and seems difficult to justify in view of the careful validation of the model on the different elementary reaction channels performed in the preceding sections, in particular when looking at the two orders of magnitude discrepancy for the lightest isotopes. Since these isotopes are also the shortest-lived ones, the responsibility of the release time can be suspected.

We have therefore corrected the production yields given by MCNPX in order to take into account a possibly rather large diffusion time. The used assumptions and derived formulas are detailed in the appendix. Since the release time is not known, different values up to 20 hours have been tried. The corrected yields are shown in figs. 11 and 12 as lines with different colors. As expected, the effect of the correction is much larger on the short-lived isotopes than on the long-lived ones but is significant even in the latter case for times above a few hours. A remarkable agreement between the calculations and the experiment is observed, regarding not only the shape of the isotopic distribution but also the absolute release rates, at both energies, for release times of the order of 10 hours. We cannot claim that the comparison of our model with the ISOLDE experimental data allows determining the astatine diffusion time. However, since we have proven in the first part of this paper that all the elementary production channels are reproduced within a factor of 2 , with

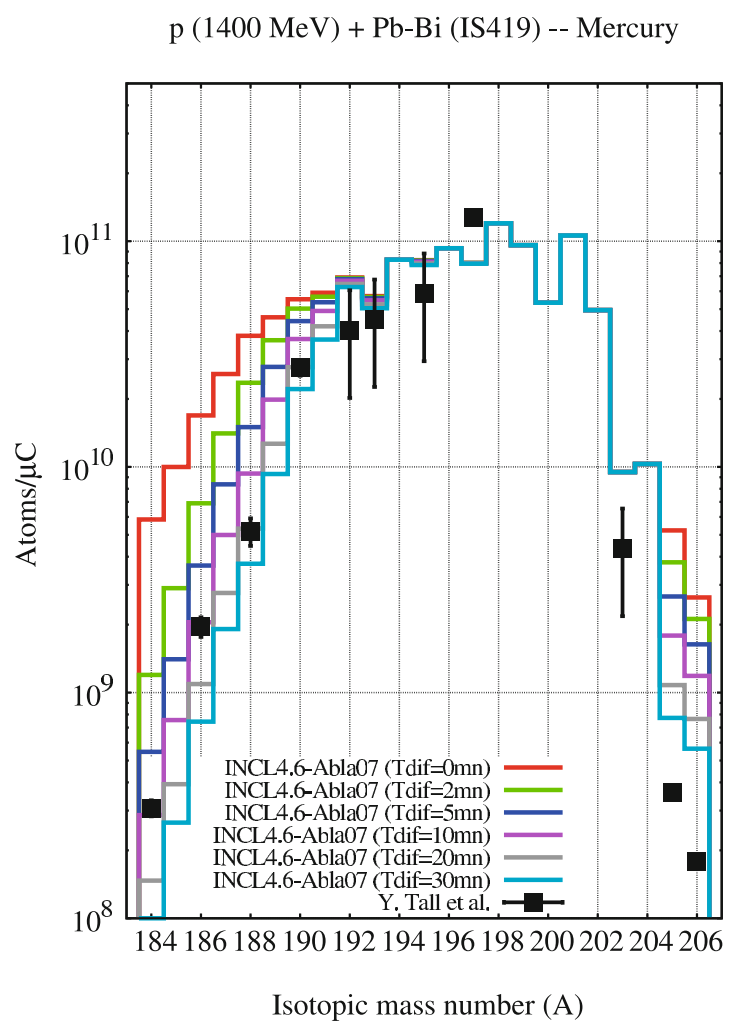

Fig. 13. Same as fig. 11 but for mercury isotopes at $1.4 \mathrm{GeV}$. Possible precursors decaying to mercury isotopes are taken into account.

a tendency to overpredict the isotopes produced by double charge exchange reactions, the comparison with the data at both incident energies seems to indicate that a diffusion time between 5 and 10 hours would be plausible. This also explains the huge discrepancy between all the models and the experimental data found in [5].

\subsection{Application to mercury isotopes}

In the same experiment, isotopes of other elements were also measured, for which the models tried in [5] give reasonable agreement with the experimental data, mainly because they are produced in less exotic channels than astatine. Some information on the diffusion time was obtained during the experiment [19]. The diffusion time was estimated by measuring the current of $\mathrm{Hg} 204+$ isotope with a Faraday cup, up to 30 minutes. At $650 \mathrm{C}$ the estimate diffusion time was of about 10 minutes. Some mercury isotopes however have a half-life shorter than this time. Therefore, we have applied our correction method to mercury to verify it. The results are shown in fig. 13 for different diffusion times.

Clearly, for the lighest isotopes, which have the shortest half-life, a diffusion time of the order of 10 to $20 \mathrm{~min}$, corresponding roughly to the estimated experimental value, greatly improves the agreement between the calculation and the experimental data. 


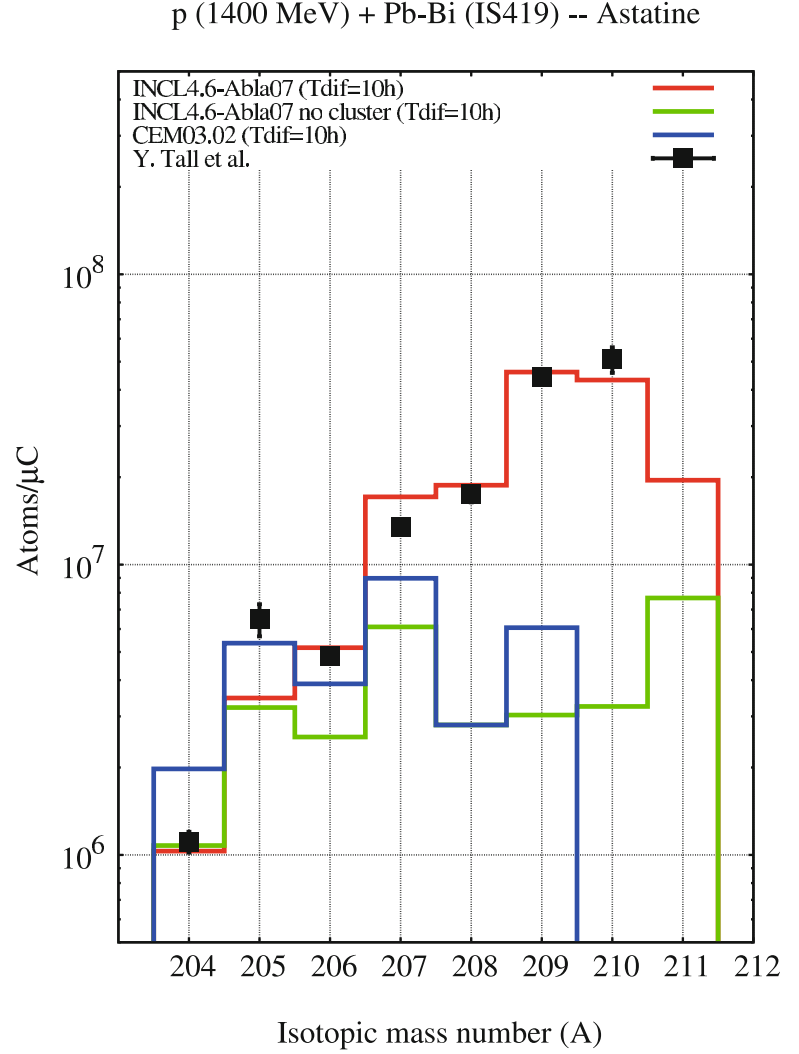

Fig. 14. Same as fig. 11, for a diffusion time of 10 hours, with the normal INCL4.6-ABLA07 compared to a calculation in which the production of clusters in the INC model was switched off (green line) and to CEM03 (blue line).

\subsection{Comparison to other models}

In order to emphasize the importance of the secondary reactions induced by the clusters produced during the cascade stage through our coalescence mechanism, a calculation has been performed switching off this mechanism. The result is presented as the green curve in fig. 14 (for a diffusion time of 10 hours) and exhibits a severe deficit of heavy isotopes. This confirms what was already suspected in sect. 4.1 looking at fig. 6. Obviously, a model that is unable to emit high-energy helium nuclei cannot be expected to correctly predict astatine production in a LBE target, since only a small fraction of the heliums produced in the evaporation stage have enough energy to undergo a reaction before being stopped. This was the case of our first version INCL4.2, which is more or less mimicked by INCL4. 6 without clusters, but also of the MCNPX default model option, Bertini-Dresner.

In fig. 14, the results are also compared with a MCNPX calculation using CEM03 [36], which actually means that the models involved are CEM03 to describe protoninduced reactions and ISABEL INC [37], the default option of MCNPX to describe reactions induced by complex particles at energies up to $1 \mathrm{GeV}$, to calculate interactions of secondary ${ }^{3} \mathrm{He}$ and ${ }^{4} \mathrm{He}$ with $\mathrm{Bi}$ (blue line). It is interesting to note that, while such a calculation more or less correctly predicts the isotope produced in double charge

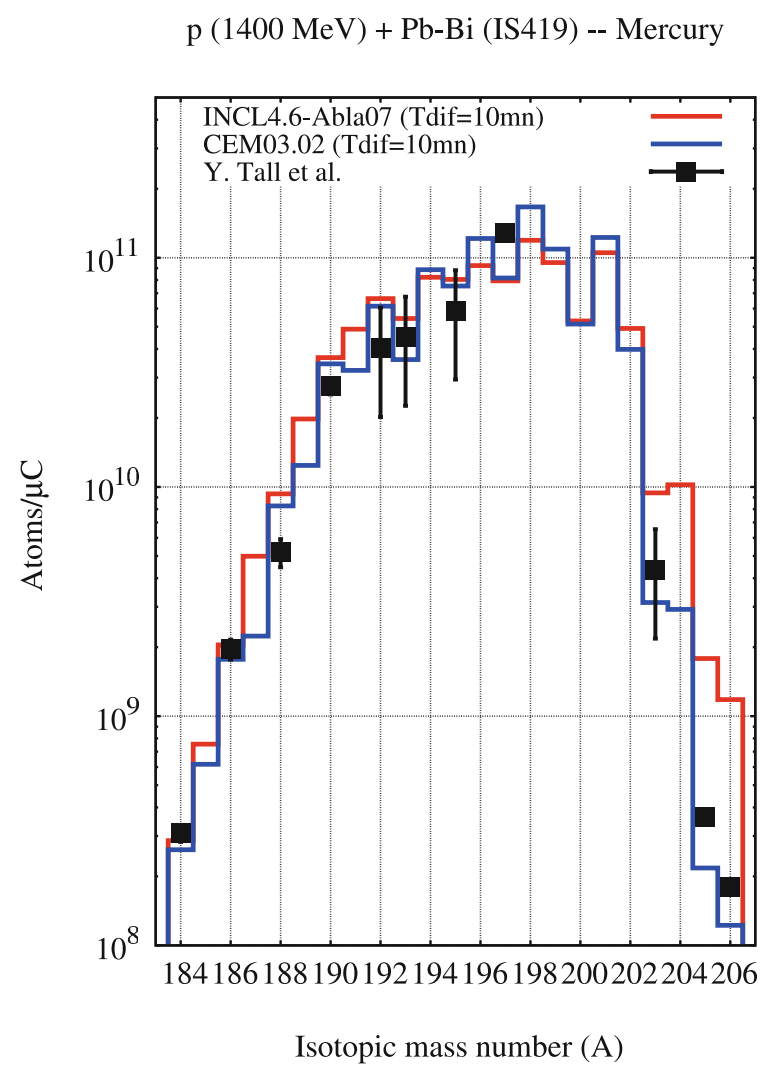

Fig. 15. Mercury isotopes measured at $1.4 \mathrm{GeV}$ compared to the INCL4.6-ABLA07 and CEM03 models for a diffusion time of $10 \mathrm{~min}$.

exchange reactions, it is not able to account for the measured yield of heavy astatine isotopes. Actually, CEM03 does have mechanisms to produce high-energy heliums, as shown in [36], but the fact that it does not produce isotopes with mass larger than 209 indicates that the problem lies probably in an inadequate treatment of low-energy helium-induced reactions by ISABEL.

We do not show here calculations corrected with different diffusion times for INCL4.6-ABLA07 without cluster and CEM03. Indeed, there is no possibility to find a diffusion time which would give a better agreement with the experimental data on the heavy isotopes without spoiling the light ones.

Results on mercury isotopes obtained by both INCL4.6-ABLA07 and CEM03 at $1.4 \mathrm{GeV}$ are displayed in fig. 15, for a diffusion time of $10 \mathrm{~min}$. It can be observed that CEM03, in this case in which secondary reactions induced by composite particles do not play any significant role, agrees very well, even better than INCL4.6, with the experimental data.

\section{Conclusion}

In this paper, we have investigated the capability of the new INCL4.6 version of the Liège intranuclear cascade model, coupled to the deexcitation code ABLA07, 
to predict astatine production in LBE spallation targets, when implemented into a high-energy transport code such as MCNPX. This study was initially motivated by the existence of experimental data from the ISOLDE Collaboration that could not be reproduced by the different codes that were tried.

We first checked that the model reasonably reproduces all the features of the elementary interactions that play a role in the generation of isotopes with a charge larger than that of the target by two units, i.e. $\left(p, \pi^{-}\right)$reactions, the energy spectrum of helium nuclei produced in primary interactions and the helium-induced secondary reactions.

With the model implemented in MCNPX, the respective contributions of the different possible reaction channels in the ISOLDE case have been identified. It showed that light astatine isotopes are mainly generated through $\left(p, \pi^{-}\right)$reactions while the heavy ones are due to secondary interactions. A detailed comparison of the model with the available experimental data from the literature for each particular channel was carried out, which gave very satisfactory results thanks in particular to improvements brought recently on the treatment of low-energy helium-induced reactions. A few remaining deficiencies were identified, as the overprediction of heavy isotopes in double charge exchange reactions and in $\left({ }^{3} \mathrm{He}, x n\right)$ reactions for low $x$-values. However, given the relatively low weight of these channels in the isotope yields in the case of the ISOLDE target, it can be said that the model has been validated and its overall accuracy estimated to be better than a factor 2 .

Finally, the full simulation of the ISOLDE experiment has been performed with the model implemented into MCNPX, taking into account a likely rather long diffusion time in the LBE target. The calculations have been compared with the measured release rates for the different astatine isotopes, at the two studied energies, 1.4 and $1 \mathrm{GeV}$. The shape of the isotopic distribution and the absolute release rates are perfectly reproduced, assuming a diffusion time between 5 and 10 hours. A simulation done switching off the cluster production through the coalescence mechanism in INCL4.6 stressed the importance of this mechanism, which produce high-energy heliums having a high probability of undergoing secondary reactions, for the astatine production. This points out that models not having a similar mechanism, such as the default Bertini-Dresner model in MCNPX, cannot be expected to reliably predict astatine in LBE. Choosing CEM03, which does have such a mechanism, in MCNPX, leads however to a large underestimation of heavy astatine isotopes. This is in fact because CEM03 (like Bertini INC) is not able to handle reactions induced by secondary heliums and other lighter fragments and MCNPX then calls by default the ISABEL INC, which has probably an inadequate treatment of lowenergy helium-induced reactions.

In conclusion, it can be said that our model, thanks to the attention paid to the emission of high-energy clusters and to low-energy cluster induced reactions, can be safely used within MCNPX to calculate the production of isotopes with a charge larger than that of the target by two units in spallation targets. In particular, it can be applied to the production of polonium isotopes in lead targets, which, for instance, was recently found in unexpected concentrations in the analyses of lead samples irradiated at the SINQ facility at PSI [38]. More generally, the model should be able to correctly predict all the isotopes created in secondary reactions induced by composite particles.

The authors would like to thank A. Guertin for valuable discussions. This work was partly supported by the FP7 Euratom project ANDES, EC contract number FP7-249671.

\section{Appendix A. Correction due to the diffusion time}

The MCNPX calculation provides the number of isotopes produced in the target per incident proton. If these isotopes are radioactive and are not immediately released, it is necessary to take into account the possible decay before release when comparing the calculation to the experiment. Let $\Phi$ be the flux of incoming beam intercepting $N_{0}$ target nuclei, $\sigma$ the average production cross-section of a given isotope, $N_{\text {prod }}$ the number of isotopes produced inside the target. The number of nuclei at a given time inside the target is called $N_{\text {in }}(t)$ and $N_{\text {out }}(t)$ is the number of nuclei that escaped from the target up to time $t$. Isotopes are supposed to decay with a half-life $T_{1 / 2}$ and are supposed to diffuse in the target before reaching the surface. The characteristic time (actually, the time of the release of half the produced quantity) for this diffusion is called $T_{d}$.

We look at the evolution of the populations in the simplest case (no geometrical effect, astatine nuclei can escape once they reach the surface, no effect of depletion of the target). We assume that astatine nuclei are detected (by their decay) as soon as they come out of the surface of the target. The irradiation starts at $t=0$ and ends at $t=t_{\text {irr }}$.

$$
\frac{\mathrm{d} N_{\text {prod }}}{\mathrm{d} t}=\Phi \sigma N_{0}
$$

We can write the Bateman equations [39]

$$
\frac{\mathrm{d} N_{i n}}{\mathrm{~d} t}=\Phi \sigma N_{0}-\frac{\ln 2}{T_{1 / 2}} N_{i n}-\frac{\ln 2}{T_{d}} N_{i n} .
$$

The diffusion proces is supposed to follow the equation

$$
\frac{\mathrm{d} N_{\text {out }}}{\mathrm{d} t}=\frac{\ln 2}{T_{d}} N_{\text {in }} .
$$

Actually, detailed studies done at ISOLDE [40] show that a more complicated process has to be considered but we want here only to estimate the order of magnitude of the correction to be applied.

The solution of eq. (A.2), for $0<t<t_{i r r}$, is

$$
N_{i n}(t)=\frac{\Phi \sigma N_{0}}{\lambda}\left(1-e^{-\lambda t}\right),
$$


where

$$
\lambda=\ln 2\left(\frac{1}{T_{d}}+\frac{1}{T_{1 / 2}}\right),
$$

Using eqs. (A.3) and (A.4), the escape rate is therefore

$$
\frac{\mathrm{d} N_{\text {out }}}{\mathrm{d} t}=\frac{\ln 2 \Phi \sigma N_{0}}{\lambda T_{d}}\left(1-e^{-\lambda t}\right) .
$$

Finally, if the measurements integrate just before the end of the irradiation time over a duration $t_{m}=t_{i r r}-t_{1}$, the measured yield per incident proton is given by

$$
Y_{\text {out }}=\frac{\int_{t_{\text {irr }}-t_{m}}^{t_{\text {ir }}} \mathrm{d} N_{\text {out }}}{\Phi t_{m}}
$$

while the production yield is

$$
Y_{\text {prod }}=\frac{\int_{t_{i r r}-t_{m}}^{t_{i r r}} \mathrm{~d} N_{\text {prod }}}{\Phi t_{m}}=\sigma N_{0} .
$$

One has

$$
Y_{\text {out }}=\frac{\ln 2 Y_{\text {prod }}}{\lambda T_{d}}\left[1+e^{-\lambda t_{\text {irr }}} \frac{1-e^{\lambda t_{m}}}{\lambda t_{m}}\right] .
$$

If $t_{m} \ll T_{1 / 2}$ and $T_{d}$, then the equation becomes

$$
Y_{\text {out }} \approx \frac{\ln 2 Y_{\text {prod }}}{\lambda T_{d}}\left[1-e^{-\lambda t_{\text {irr }}}\right] .
$$

For the limit of $T_{1 / 2} \ll T_{d}, t_{i r r}$,

$$
\lambda \approx \frac{\ln 2}{T_{1 / 2}}
$$

and then

$$
Y_{\text {out }} \approx \frac{Y_{\text {prod }} T_{1 / 2}}{T_{d}}
$$

In our case, astatine isotopes have half-lifes between 9 minutes and 8 hours, the measurement is done during a short time (a few seconds) after a rather long time of irradiation, $t_{i r r}$ of the order of 22 hours and we have considered diffusion times between several tens of minutes to 20 hours. Therefore, we can use most of the time eq. (A.10).

In the case of mercury, generally $T_{d} \ll T_{1 / 2}$ and $t_{i r r}$, so $\lambda \approx \frac{\ln 2}{T_{d}}$ and then $Y_{\text {out }} \approx Y_{\text {prod }}$ except for the isotopes with the shortest periods.

\section{References}

1. E. Kugler, Hyperfine Interact. 129, 23 (2000).

2. G.S. Bauer et al., J. Nucl. Mat. 296, 17 (2001).

3. H. Abderrahim, P. Baeten, D. De Bruyn, J. Heyse, P. Schuurmans, J. Wagemans, Nucl. Phys. News 20, 24 (2010).

4. L. Zanini et al., in International Conference on Nuclear Data for Science and Technology, edited by R.C. Haight et al. (Melville, New York, 2005) p. 1525.
5. Y. Tall et al., Proceedings of the International Conference on Nuclear Data for Science and Technology, April 22-27, 200\%, Nice, France, edited by O. Bersillon, F. Gunsing, E. Bauge, R. Jacqmin, S. Leray (EDP Sciences, 2008) p. 1069.

6. A. Fassò, A. Ferrari, J. Ranft, P.R. Sala, FLUKA: a multiparticle transport code, CERN-2005-10, INFN/TC_05/11 (2005).

7. J.S. Hendricks et al., "MCNPX EXTENSIONS VERSION 2.5.0", Los Alamos National Laboratory Report LA-UR05-2675 (2005).

8. A. Boudard, J. Cugnon, S. Leray, C. Volant, Phys. Rev. C 66, 044615 (2002).

9. A.R. Junghans et al., Nucl. Phys. A 629, 635 (1998).

10. H.W. Bertini, Phys. Rev. 131, 1801 (1963).

11. L. Dresner, Oak Ridge report, Technical report, ORNLTM-196, 1962.

12. A. Boudard, J. Cugnon, in Proceedings of the Joint ICTPIAEA Advanced Workshop on Model Codes for Spallation Reactions, ICTP Trieste, Italy, 4-8 February 2008, edited by D. Filges, S. Leray, Y. Yariv, A. Mengoni, A. Stanculescu, G. Mank (IAEA INDC(NDS)-530, Vienna, 2008) p. 29, http://www-nds.iaea.org/reports-new/indcreports/indc-nds/indc-nds-0530.pdf.

13. A. Kelic, M.V. Ricciardi, K.-H. Schmidt, in Proceedings of the Joint ICTP-IAEA Advanced Workshop on Model Codes for Spallation Reactions, ICTP Trieste, Italy, 4-8 February 2008, edited by D. Filges, S. Leray, Y. Yariv, A. Mengoni, A. Stanculescu, G. Mank (IAEA INDC(NDS)-530, Vienna, 2008) p. 181, http:// www-nds.iaea.org/reports-new/indc-reports/indcnds/indc-nds-0530.pdf.

14. FP6 Euratom project EUROTRANS/NUDATRA, EC contract number FI6W-CT-2004-516520.

15. http://www-nds.iaea.org/spallations.

16. S. Leray et al., J. Korean Phys. Soc. 59, 791 (2011).

17. A. Boudard et al., Phys. Rev. C. 87, 014606 (2013).

18. J.C. David, J.S. Hendricks, private communication.

19. Y. Tall, PhD Thesis, Université de Nantes, France (2008).

20. A. Boudard, J. Cugnon, S. Leray, C. Volant, Nucl. Phys. A 740, 195 (2004).

21. Th. Aoust, J. Cugnon, Phys. Rev. C 74, 064607 (2006).

22. D.R.F. Cochran et al., Phys. Rev. D 6, 3085 (1972).

23. C.M. Herbach et al., Nucl. Phys. A 765, 426 (2006).

24. S. Leray et al., Nucl. Instrum. Methods Phys. Res. B 268, $581(2010)$.

25. G. Igo, B.D. Wilkins, Phys. Rev. 131, 1251 (1963).

26. A. Ingemarsson et al., Nucl. Phys. A 676, 3 (2000).

27. A. Ingemarsson et al., Nucl. Phys. A 696, 3 (2001).

28. M. Dombsky et al., Phys. Rev. C 32, 253 (1985).

29. P. Napolitani et al., Phys. Rev. C 76, 064609 (2007).

30. http://www-nds.iaea.org/exfor/.

31. R.J. Charity, in Proceedings of the Joint ICTP-IAEA Advanced Workshop on Model Codes for Spallation Reactions, ICTP Trieste, Italy, 4-8 February 2008, edited by D. Filges, S. Leray, Y. Yariv, A. Mengoni, A. Stanculescu, G. Mank (IAEA INDC(NDS)-530, Vienna, 2008) p. 139, http://www-nds .iaea.org/reports-new/indc-reports/ indc-nds/indc-nds-0530.pdf.

32. D. Mancusi, R.J. Charity, J. Cugnon, Phys. Rev. C 82, 084610 (2010).

33. D. Mancusi, private communication.

34. N.E. Scott, J.W. Cobble, P.J. Daly, Nucl. Phys. A 119, 131 (1968). 
35. L. Zanini et al., in preparation.

36. S.G. Mashnik et al., J. Phys.: Conf. Ser. 41, 340 (2006).

37. Y. Yariv, Z. Fraenkel, Phys. Rev. C 20, 2227 (1979).
38. D. Schumann, private communication.

39. H. Bateman, Proc. Cambridge Philos. Soc. 15, 423 (1910).

40. M. Turrion et al., Nucl. Instrum. Methods Phys. Res. B 266, 4674 (2008). 